\title{
Outage Probability of Dual-Hop Multiple Antenna Relay Systems with Interference at the Relay and Destination
}

\author{
Mengmeng Liu, ${ }^{1}$ Jianhua Zhang, ${ }^{2}$ and Ping Zhang' \\ ${ }^{1}$ State Key Laboratory of Networking and Switching Technology, Beijing University of Posts and Telecommunications, \\ Beijing 100876, China \\ ${ }^{2}$ Key Laboratory of Universal Wireless Communications, Ministry of Education, Beijing University of Posts and Telecommunications, \\ Beijing 100876, China
}

Correspondence should be addressed to Mengmeng Liu; liumm.bupt@hotmail.com

Received 6 August 2014; Revised 1 October 2014; Accepted 9 October 2014; Published 27 October 2014

Academic Editor: Feifei Gao

Copyright (C) 2014 Mengmeng Liu et al. This is an open access article distributed under the Creative Commons Attribution License, which permits unrestricted use, distribution, and reproduction in any medium, provided the original work is properly cited.

This paper analyzes the outage performance of a dual-hop relaying system in which the relay is equipped with multiple antennas, while the source and destination have a single antenna. New exact closed-form expressions for the outage probability of both the amplify-and-forward (AF) and the decode-and-forward (DF) relaying systems are derived, assuming that the relay and destination are impaired by cochannel interferers and additive white Gaussian noise (AWGN). Numerical results are presented to verify the theoretical analysis.

\section{Introduction}

Relaying methodologies such as amplify-and-forward (AF) and decode-and-forward (DF) have received considerable interest by virtue of improving the range and link reliability in fading wireless channels. Meanwhile, the more aggressive frequency reuse strategy results in increasingly complex interference environment. Several recent works have studied the impact of cochannel interference (CCI) on the outage performance of the relaying system when the relay or/and destination are subjected to single or multiple cochannel interferers. In [1], the effect of multiple Rayleigh fading interferers in an AF relay system was investigated and exact expression for outage probability was derived. Reference [2] also studied the outage probability of a fixed gain AF relaying system under the influence of interference at the relay and the destination terminals. However, $[1,2]$ only considered a single antenna system, which is one common limitation in most of the prior literatures.

Despite the importance of multiple-input multipleoutput (MIMO) technology, few papers have studied the performance of multiple antenna relaying systems in the presence of CCI. For example, [3] derived an exact closed-form expression for the outage probability of a dual-hop AF MIMO relay network where the source and destination have multiple antennas and the relay has only one antenna, while [4] addressed the case where only one of the nodes is equipped with multiple antennas and the relay node is subjected to a single interferer, and later [5] extended [4] to systems with arbitrary number of interferers at the relay node, assuming that the relay is subjected to CCI and additive white Gaussian noise (AWGN) while the destination is corrupted by AWGN only. In [6], the outage expressions for different configurations of the multiple antenna relay network with interference in the context of AF relay with fixed gain were derived. Most recently, [7] proposed a two-hop AF relaying scheme considering a system with CCI and thermal noise at both the relay and destination, and [8] investigated the outage probability of an AF relay system in which the source is equipped with multiple transmit antennas and adopts the orthogonal spacetime block code to increase the system performance.

Unlike the aforementioned works, this paper aims to analyze the outage performance of a dual-hop multiple antenna relay system with arbitrary number of cochannel interferers at both the relay and the destination along with thermal noise. For the reason of mathematical tractability, we neglect 


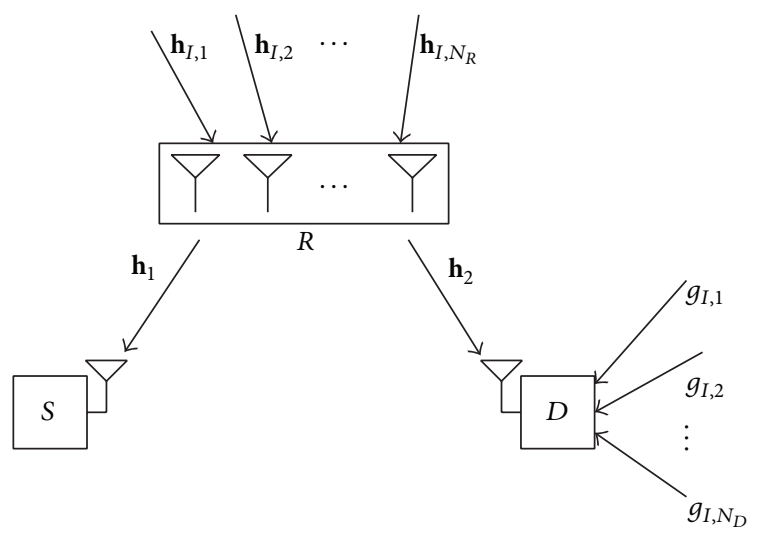

Figure 1: System diagram.

the effect of noise in AF scheme. The main contribution is the derivation of new exact closed-form expressions for the outage probability of the system under consideration employing either an AF or a DF relay.

\section{System Models}

Consider a dual-hop relaying system, where the source $S$ and destination $D$ have only one antenna while the relay $R$ is equipped with $N$ antennas, as illustrated in Figure 1. It is assumed that both the relay and destination suffer from CCI and AWGN. All the channel coefficients are independent and identically distributed (i.i.d.) complex Gaussian random variables with zero mean and unit variance. Besides, there is no direct link between the source and destination due to obstacles or deep fading.

Each transmission consists of two phases. In the first phase, the source transmits the signal to the relay, and the received vector at the relay is given by

$$
\mathbf{y}_{r}=\mathbf{h}_{1} x+\sum_{i=1}^{N_{R}} \mathbf{h}_{I, i} s_{R, i}+\mathbf{n}_{r}
$$

where the $N \times 1$ vectors $\mathbf{h}_{1}$ and $\mathbf{h}_{I, i}$ denote the channel from the source and the $i$ th interferer, respectively. $N_{R}$ is the number of interferers at the relay node, $x$ is the information symbol satisfying $\mathbb{E}\left\{|x|^{2}\right\}=P_{s}, s_{R, i}$ is the $i$ th interference symbol with $\mathbb{E}\left\{\left|s_{R, i}\right|^{2}\right\}=P_{R, i}$, and $\mathbf{n}_{r} \sim \mathscr{C} \mathscr{N}\left(0, \sigma_{r}^{2} \mathbf{I}\right)$ represents the AWGN at the relay.

In the second phase, an AF relay retransmits a transformed version of the received signal to the destination, and the signal at the destination is expressed as

$$
y_{d}^{\mathrm{AF}}=\mathbf{h}_{2} \mathbf{W y}_{r}+\sum_{j=1}^{N_{D}} g_{I, j} s_{D, j}+n_{d},
$$

where $\mathbf{h}_{2} \in \mathbb{C}^{1 \times N}$ and $g_{I, j}$ denote the channel from the relay and the $j$ th interferer, respectively. $N_{D}$ is the number of interferers at the destination, $s_{D, j}$ is the $j$ th interference symbol with $\mathbb{E}\left\{\left|s_{D, j}\right|^{2}\right\}=P_{D, j}, n_{d}$ is the AWGN with
$\mathbb{E}\left\{\left|n_{d}\right|^{2}\right\}=\sigma_{d}^{2}$, and $\mathbf{W}$ is the $N \times N$ transformation matrix and will be specified in the following section.

As a result, the instantaneous signal-to-interference-plusnoise ratio (SINR) experienced at the destination is given by

$$
\gamma_{d}^{\mathrm{AF}}=\frac{\left|\mathbf{h}_{2} \mathbf{W} \mathbf{h}_{1}\right|^{2} P_{s}}{\sum_{i=1}^{N_{R}}\left|\mathbf{h}_{2} \mathbf{W h}_{I, i}\right|^{2} P_{R, i}+\left\|\mathbf{h}_{2} \mathbf{W}\right\|^{2} \sigma_{r}^{2}+z_{d}} .
$$

Here, $z_{d}=\sum_{j=1}^{N_{D}}\left|g_{I, j}\right|^{2} P_{D, j}+\sigma_{d}^{2}$ denotes the undesired interference-plus-noise signal received at the destination. For a given threshold value $\gamma_{\text {th }}$, the outage probability of the AF relaying system can be mathematically expressed as

$$
P_{\text {out }}^{\mathrm{AF}}=P\left(\gamma_{d}^{\mathrm{AF}}<\gamma_{\mathrm{th}}\right) \text {. }
$$

On the other hand, when the DF protocol is adopted, the relay first decodes the received signal and then forwards the reencoded message $s_{r}$ to the destination with $\mathbb{E}\left\{\left|s_{r}\right|^{2}\right\}=P_{s_{r}}$. We consider two cases: (1) the DF relay applies some sort of transformation to the message $s_{r}$; (2) the DF relay does not perform any linear diversity combining. In the first case, the received signal at the destination is

$$
y_{d}^{\mathrm{DF}, 1}=\mathbf{h}_{2} \mathbf{W} s_{r}+\sum_{j=1}^{N_{D}} g_{I, j} s_{D, j}+n_{d}
$$

where $\mathbf{W}$ is the $N \times 1$ precoding matrix. The SINR at the destination is given by

$$
\gamma_{d}^{\mathrm{DF}, 1}=\frac{\left|\mathbf{h}_{2} \mathbf{W}\right|^{2} P_{s_{r}}}{\sum_{j=1}^{N_{D}}\left|g_{I, j}\right|^{2} P_{D, j}+\sigma_{d}^{2}} .
$$

The outage probability of the DF relaying system can be expressed as

$$
P_{\mathrm{out}}^{\mathrm{DF}, 1}=P\left(\gamma_{d}^{\mathrm{DF}, 1}<\gamma_{\mathrm{th}}\right)
$$

For the second case, the received signal at the destination becomes

$$
y_{d}^{\mathrm{DF}, 2}=h_{2} s_{r}+\sum_{j=1}^{N_{D}} g_{I, j} s_{D, j}+n_{d} .
$$

Then, the outage probability of the DF relaying system can be defined as

$$
P_{\text {out }}^{\mathrm{DF}, 2}=P\left(\min \left\{\max \left\{\gamma_{r, k}^{\mathrm{DF}, 2}\right\}, \gamma_{d}^{\mathrm{DF}, 2}\right\}<\gamma_{\mathrm{th}}\right),
$$

where $\gamma_{r, k}^{\mathrm{DF}, 2}$ and $\gamma_{d}^{\mathrm{DF}, 2}$ represent the SINR at the $k$ th relay antenna and destination, respectively. Let $h_{1, k}$ and $h_{I, i, k}$ be the $k$ th element of $\mathbf{h}_{1}$ and $\mathbf{h}_{I, i}$; from (1), the received signal at the $k$ th relay antenna is given by

$$
y_{r, k}=h_{1, k} x+\sum_{i=1}^{N_{R}} h_{I, i, k} s_{R, i}+n_{r, k} .
$$


Thus, we have

$$
\begin{gathered}
\gamma_{r, k}^{\mathrm{DF}, 2}=\frac{\left|h_{1, k}\right|^{2} P_{s}}{\sum_{i=1}^{N_{R}}\left|h_{I, i, k}\right|^{2} P_{R, i}+\sigma_{r}^{2}}, \quad k=1, \ldots, N, \\
\gamma_{d}^{\mathrm{DF}, 2}=\frac{\left|h_{2}\right|^{2} P_{r}}{\sum_{j=1}^{N_{D}}\left|g_{I, j}\right|^{2} P_{D, j}+\sigma_{d}^{2}} .
\end{gathered}
$$

\section{Outage Probability Analysis}

In this section, we derive new exact analytical expressions for the outage probability of the considered system. For mathematical tractability, if AF protocol is adopted, the relay and destination are assumed to be interference-limited; that is, the effect of the noise at the relay and destination can be neglected.

3.1. AF Relay Systems. Since the optimal relay precoder matrix is analytically intractable, although performing maximum ratio combining will not minimize CCI, we still use a two-stage relay processing strategy, that is, the maximum ratio combining/maximal ratio transmission (MRC/MRT) scheme [5]. Therefore, the relay transformation matrix $\mathbf{W}$ can be written as $\mathbf{W}=G\left(\mathbf{h}_{2}^{\dagger} \mathbf{h}_{1}^{\dagger} /\left\|\mathbf{h}_{2}\right\|\left\|\mathbf{h}_{1}\right\|\right)$. In order to satisfy the transmit power constraint of the relay, that is, $\mathbb{E}\left\{\left\|\mathbf{W y}_{r}\right\|^{2}\right\}=$ $P_{r}, G$ can be chosen as

$$
G=\sqrt{\frac{P_{r}}{P_{s} N+\sum_{i=1}^{N_{R}} P_{R, i}+\sigma_{r}^{2}}}
$$

Substituting $\mathbf{W}$ into (3) and after some derivations, $\gamma_{d}^{\mathrm{AF}}$ can be expressed as

$$
\begin{aligned}
& \gamma_{d}^{\mathrm{AF}} \\
& =\frac{\left\|\mathbf{h}_{2}\right\|^{2}\left\|\mathbf{h}_{1}\right\|^{2} P_{s}}{\left\|\mathbf{h}_{2}\right\|^{2} \sum_{i=1}^{N_{R}}\left(\left|\mathbf{h}_{1}^{\dagger} \mathbf{h}_{I, i}\right|^{2} /\left\|\mathbf{h}_{1}\right\|^{2}\right) P_{R, i}+\left\|\mathbf{h}_{2}\right\|^{2} \sigma_{r}^{2}+z_{d}\left(1 / G^{2}\right)} .
\end{aligned}
$$

For notational convenience, we define $\gamma_{1}=\left(P_{s} / \sigma_{r}^{2}\right)\left\|\mathbf{h}_{1}\right\|^{2}, \gamma_{2}=$ $\left(P_{r} / \sigma_{d}^{2}\right)\left\|\mathbf{h}_{2}\right\|^{2}, \gamma_{R}=\sum_{i=1}^{N_{R}}\left(P_{R, i} / \sigma_{r}^{2}\right) h_{R, i}, h_{R, i}=\left|\mathbf{h}_{1}^{\dagger} \mathbf{h}_{I, i}\right|^{2} /\left\|\mathbf{h}_{1}\right\|^{2}$ and $\gamma_{D}=\sum_{j=1}^{N_{D}}\left(P_{D, j} / \sigma_{d}^{2}\right)\left|g_{I, j}\right|^{2}$. Since the relay and destination are interference-limited, $\gamma_{d}^{\mathrm{AF}}$ can be further derived as

$$
\gamma_{d}^{\mathrm{AF}}=\frac{\gamma_{1} \gamma_{2}}{\gamma_{2} \gamma_{R}+C \gamma_{D}}
$$

where the constant $C=\left(P_{s} / \sigma_{r}^{2}\right) N+\sum_{i=1}^{N_{R}}\left(P_{R, i} / \sigma_{r}^{2}\right)+1$.

To evaluate the outage performance, the probability density function (PDF) of $\gamma_{1}, \gamma_{2}, \gamma_{R}$, and $\gamma_{D}$ is required. It is easy to observe that $\gamma_{1}$ and $\gamma_{2}$ are i.i.d. random variables with PDF

$$
f_{\gamma_{i}}(\gamma)=\frac{1}{\rho_{i}^{N}(N-1) !} \gamma^{N-1} e^{-\gamma / \rho_{i}} U(\gamma),
$$

where $\rho_{1}=P_{s} / \sigma_{r}^{2}, \rho_{2}=P_{r} / \sigma_{d}^{2}$, and $U(\cdot)$ is the unit-step function.

Given that $\left\{h_{R, i}\right\}_{i=1}^{N_{R}}$ are i.i.d. exponential random variables with unit variance, then, $\gamma_{R}$ follows the hyperexponential distribution with PDF $[1,5]$ as

$$
f_{\gamma_{R}}(\gamma)=\sum_{m=1}^{\varrho(\mathscr{A})} \sum_{n=1}^{\tau_{m}(\mathscr{A})} \mathscr{X}_{m, n}(\mathscr{A}) \frac{\rho_{R,[m]}^{-n}}{(n-1) !} \gamma^{n-1} e^{-\gamma / \rho_{R,[m]}} U(\gamma),
$$

where $\mathscr{A}=\operatorname{diag}\left(\rho_{R, 1}, \ldots, \rho_{R, N_{R}}\right), \rho_{R, i}=P_{R, i} / \sigma_{r}^{2}, \varrho(\mathscr{A})$ denotes the number of distinct diagonal elements of $\mathscr{A},\left\{\rho_{R,[m]}\right\}$ are the distinct diagonal elements in decreasing order, $\tau_{m}(\mathscr{A})$ is the multiplicity of $\rho_{R,[m]}$, and $\mathscr{X}_{m, n}(\mathscr{A})$ is the $(m, n)$ th characteristic coefficient of $\mathscr{A}$. Similarly, the PDF of $\gamma_{D}$ is given by

$$
f_{\gamma_{D}}(\gamma)=\sum_{m=1}^{\varrho(\mathscr{B})} \sum_{n=1}^{\tau_{m}(\mathscr{B})} \mathscr{X}_{m, n}(\mathscr{B}) \frac{\rho_{D,[m]}^{-n}}{(n-1) !} \gamma^{n-1} e^{-\gamma / \rho_{D,[m]}} U(\gamma)
$$

where $\mathscr{B}=\operatorname{diag}\left(\rho_{D, 1}, \ldots, \rho_{D, N_{D}}\right), \rho_{D, j}=P_{D, j} / \sigma_{d}^{2}, \varrho(\mathscr{B})$, $\tau_{m}(\mathscr{B})$, and $\mathscr{X}_{m, n}(\mathscr{B})$ are defined the same as $(16)$.

Combining (4) with (14) and using the PDF of $\gamma_{1}$ given by (15), $P_{\text {out }}^{\mathrm{AF}}$ can be formulated as (18), where the last equality of (18) is obtained by using the binomial expansion

$$
\begin{aligned}
& P_{\text {out }}^{\mathrm{AF}} \\
& =P\left(\gamma_{1}<\gamma_{\mathrm{th}} \gamma_{R}+\gamma_{\mathrm{th}} C \frac{\gamma_{D}}{\gamma_{2}}\right) \\
& =1-\sum_{p=0}^{N-1} \frac{1}{p !} \iiint_{0}^{\infty}\left(\frac{\gamma_{\mathrm{th}} \gamma_{R}}{\rho_{1}}+\frac{\gamma_{\mathrm{th}} C}{\rho_{1}} \frac{\gamma_{D}}{\gamma_{2}}\right)^{p} \\
& \times e^{-\left(\gamma_{\mathrm{th}} / \rho_{1}\right) \gamma_{R}} e^{-\left(\gamma_{\mathrm{th}} C / \rho_{1}\right)\left(\gamma_{D} / \gamma_{2}\right)} f_{\gamma_{2}}\left(\gamma_{2}\right) \\
& \times f_{\gamma_{R}}\left(\gamma_{R}\right) f_{\gamma_{D}}\left(\gamma_{D}\right) d \gamma_{2} d \gamma_{R} d \gamma_{D} \\
& =1-\sum_{p=0}^{N-1} \sum_{q=0}^{p} \frac{1}{p !}\left(\begin{array}{l}
p \\
q
\end{array}\right)\left(\frac{\gamma_{\mathrm{th}}}{\rho_{1}}\right)^{p} C^{q} \\
& \times \underbrace{\int_{0}^{\infty} \gamma_{R}^{p-q} e^{-\left(\gamma_{\mathrm{th}} / \rho_{1}\right) \gamma_{R}} f_{\gamma_{R}}\left(\gamma_{R}\right) d \gamma_{R}}_{I_{1}} \\
& \times \underbrace{\iint_{0}^{\infty}\left(\frac{\gamma_{D}}{\gamma_{2}}\right)^{q} e^{-\left(\gamma_{\mathrm{th}} C / \rho_{1}\right)\left(\gamma_{D} / \gamma_{2}\right)} f_{\gamma_{2}}\left(\gamma_{2}\right) f_{\gamma_{D}}\left(\gamma_{D}\right) d \gamma_{2} d \gamma_{D}}_{I_{2}}
\end{aligned}
$$


Substituting the PDF of $\gamma_{R}$ given by (16) into $I_{1}$, with the help of [9, Equation (3.351.3)], $I_{1}$ can be derived as

$$
I_{1}=\sum_{m=1}^{\varrho(\mathscr{A})} \sum_{n=1}^{\tau_{m}(\mathscr{A})} \mathscr{X}_{m, n}(\mathscr{A}) \rho_{R,[m]}^{-n} \frac{(\alpha-1) !}{(n-1) !}\left(\frac{\gamma_{\mathrm{th}}}{\rho_{1}}+\frac{1}{\rho_{R,[m]}}\right)^{-\alpha},
$$

where $\alpha=n+p-q$.

To calculate the integral of $I_{2}$, we first substitute the PDF of $\gamma_{2}$ given by (15) into $I_{2}$ and then use the integral identity [9, Equation (3.471.9)] to obtain

$$
\begin{aligned}
I_{2}=\frac{2}{\rho_{2}^{N}(N-1) !} \int_{0}^{\infty} \gamma_{D}^{q}\left(\frac{\gamma_{\mathrm{th}} C \rho_{2} \gamma_{D}}{\rho_{1}}\right)^{(N-q) / 2} \\
\quad \times K_{N-q}\left(2 \sqrt{\frac{\gamma_{\mathrm{th} C \gamma_{D}}}{\rho_{1} \rho_{2}}}\right) f_{\gamma_{D}}\left(\gamma_{D}\right) d \gamma_{D}
\end{aligned}
$$

where $K_{n}(z)$ is the $n$th order modified Bessel function of the second kind [9, Equation (8.407)].

Substituting (17) into (20) and using [9, Equation (6.631.3)], after some algebraic manipulation, we get

$$
\begin{aligned}
I_{2}= & \frac{1}{(N-1) !}\left(\frac{\gamma_{\mathrm{th}} C}{\rho_{1}}\right)^{a} \rho_{2}^{-b} \sum_{m=1}^{\varrho(\mathscr{B})} \sum_{n=1}^{\tau_{m}(\mathscr{B})} \mathscr{X}_{m, n}(\mathscr{B}) \frac{\rho_{D,[m]}^{b}}{(n-1) !} \\
& \times \Gamma(n+N) \Gamma(n+q) \exp \left(\frac{\bar{\rho}_{m}}{2}\right) W_{-(n+b),(N-q) / 2}\left(\bar{\rho}_{m}\right),
\end{aligned}
$$

where $a=(N-q-1) / 2, b=(N+q-1) / 2$, $\bar{\rho}_{m}=\gamma_{\mathrm{th}} C \rho_{D,[m]} / \rho_{1} \rho_{2}$, and $W_{\lambda, \mu}(z)$ is the Whittaker function defined in [9, Equation (9.222.1)].

Now substituting (19) and (21) into (18), the closed-form expression for the outage probability of the AF relay system can be obtained as

$$
\begin{aligned}
& P_{\text {out }}^{\mathrm{AF}} \\
& =1-\frac{1}{(N-1) !} \sum_{p=0}^{N-1} \sum_{q=0}^{p} \frac{1}{p !}\left(\begin{array}{c}
p \\
q
\end{array}\right)\left(\frac{\gamma_{\mathrm{th}}}{\rho_{1}}\right)^{p+a}\left(\frac{C}{\rho_{2}}\right)^{b} \\
& \quad \times \sum_{m=1}^{\varrho(\mathscr{A})} \sum_{n=1}^{\tau_{m}(\mathscr{A})} \mathscr{X}_{m, n}(\mathscr{A}) \rho_{R,[m]}^{-n} \frac{(\alpha-1) !}{(n-1) !}\left(\frac{\gamma_{\mathrm{th}}}{\rho_{1}}+\frac{1}{\rho_{R,[m]}}\right)^{-\alpha} \\
& \quad \times \sum_{u=1}^{\varrho(\mathscr{B}) \tau_{u=1}(\mathscr{B})} \mathscr{X}_{u, v}(\mathscr{B}) \frac{\rho_{D,[u]}^{b}}{(v-1) !}(v+N) \Gamma(v+q) \\
& \quad \times \exp \left(\frac{\bar{\rho}_{u}}{2}\right) \\
& \quad \times W_{-(v+b),(N-q) / 2}\left(\bar{\rho}_{u}\right) .
\end{aligned}
$$

For the case of equal-power interference, that is, $\rho_{R, i}=\rho_{R}$, $\forall i=1, \ldots, N_{R}, \rho_{D, j}=\rho_{D}, \forall j=1, \ldots, N_{D},(22)$ reduces to

$$
\begin{aligned}
P_{\text {out }}^{\mathrm{AF}}= & 1-\frac{1}{(N-1) !} \sum_{p=0}^{N-1} \sum_{q=0}^{p} \frac{1}{p !}\left(\begin{array}{l}
p \\
q
\end{array}\right)\left(\frac{\gamma_{\mathrm{th}}}{\rho_{1}}\right)^{p+a}\left(\frac{C}{\rho_{2}}\right)^{b} \\
& \times \rho_{R}^{-N_{R}} \frac{\left(N_{R}+p-q-1\right) !}{\left(N_{R}-1\right) !}\left(\frac{\gamma_{\mathrm{th}}}{\rho_{1}}+\frac{1}{\rho_{R}}\right)^{-N_{R}-p+q} \\
& \times \frac{\rho_{D}^{b}}{\left(N_{D}-1\right) !} \Gamma\left(N_{D}+N\right) \Gamma\left(N_{D}+q\right) \exp \left(\frac{\bar{\rho}_{u}}{2}\right) \\
& \times W_{-\left(N_{D}+b\right),(N-q) / 2}\left(\bar{\rho}_{u}\right) .
\end{aligned}
$$

3.2. DF Relay Systems. In the analysis of DF relay channels, the relay and destination are assumed to be interference-andnoise-limited, and the effect of noise is taken into account. We consider two cases, respectively. In the first case, we use the maximal ratio transmission scheme and the relay precoding matrix $\mathbf{W}$ is set to be $\mathbf{W}=G\left(\mathbf{h}_{2}^{\dagger} /\left\|\mathbf{h}_{2}\right\|\right)$. In order to meet the relay power constraint $\mathbb{E}\left\{\left\|\mathbf{W} s_{r}\right\|^{2}\right\}=P_{r}, G$ can be chosen as $G=\sqrt{P_{r} / P_{s_{r}}}$. After some substitutions, (6) becomes

$$
\gamma_{d}^{\mathrm{DF}, 1}=\frac{\gamma_{2}}{\gamma_{D}+1}
$$

Then,

$$
\begin{aligned}
P_{\text {out }}^{\mathrm{DF}, 1}= & P\left(\frac{\gamma_{2}}{\gamma_{D}+1}<\gamma_{\mathrm{th}}\right)=P\left(\gamma_{2}<\gamma_{\mathrm{th}}\left(\gamma_{D}+1\right)\right) \\
= & 1-\sum_{p=0}^{N-1} \frac{1}{p !} \int_{0}^{\infty}\left(\frac{\gamma_{\mathrm{th}}\left(\gamma_{D}+1\right)}{\rho_{2}}\right)^{p} e^{-\gamma_{\mathrm{th}}\left(\gamma_{D}+1\right) / \rho_{2}} \\
& \times f_{\gamma_{D}}\left(\gamma_{D}\right) d \gamma_{D} \\
= & 1-\sum_{p=0}^{N-1} \sum_{q=0}^{p} \frac{1}{p !}\left(\begin{array}{l}
p \\
q
\end{array}\right)\left(\frac{\gamma_{\text {th }}}{\rho_{2}}\right)^{p} e^{-\gamma_{\mathrm{th}} / \rho_{2}} I_{3},
\end{aligned}
$$

where $I_{3}=\int_{0}^{\infty} \gamma_{D}^{p-q} e^{-\left(\gamma_{\mathrm{th}} / \rho_{2}\right) \gamma_{D}} f_{\gamma_{D}}\left(\gamma_{D}\right) d \gamma_{D}$. Similar to the integral of $I_{1}$ in (18), we obtain $P_{\text {out }}^{\mathrm{DF}, 1}$ as follows:

$$
\begin{aligned}
P_{\mathrm{out}}^{\mathrm{DF}, 1}= & 1-\sum_{p=0}^{N-1} \sum_{q=0}^{p} \frac{1}{p !}\left(\begin{array}{c}
p \\
q
\end{array}\right)\left(\frac{\gamma_{\mathrm{th}}}{\rho_{2}}\right)^{p} \\
& \times e^{-\gamma_{\mathrm{th}} / \rho_{2}} \sum_{m=1}^{\varrho(\mathscr{B})} \sum_{n=1}^{\tau_{m}(\mathscr{B})} x_{m, n}(\mathscr{B}) \rho_{D,[m]}^{-n} \\
& \times \frac{(\alpha-1) !}{(n-1) !}\left(\frac{\gamma_{\mathrm{th}}}{\rho_{2}}+\frac{1}{\rho_{D,[m]}}\right)^{-\alpha} .
\end{aligned}
$$


For the second case, we first define $\gamma_{1, k}=\rho_{1}\left|h_{1, k}\right|^{2}, \gamma_{2}^{\mathrm{DF}}=$ $\rho_{2}\left|h_{2}\right|^{2}$, and $\gamma_{R, k}=\sum_{i=1}^{N_{R}} \rho_{R, i}\left|h_{I, i, k}\right|^{2}$. As a consequence, the SINR in (11) can be written as

$$
\begin{gathered}
\gamma_{r, k}^{\mathrm{DF}, 2}=\frac{\gamma_{1, k}}{\gamma_{R, k}+1}, \quad k=1, \ldots, N, \\
\gamma_{d}^{\mathrm{DF}, 2}=\frac{\gamma_{2}^{\mathrm{DF}}}{\gamma_{D}+1} .
\end{gathered}
$$

Here, $\gamma_{1, k}$ and $\gamma_{2}^{\mathrm{DF}}$ are exponentially distributed with parameters $\rho_{1}$ and $\rho_{2}$, respectively, and $\gamma_{R, k}$ is distributed the same as $\gamma_{R}$ defined in (16).

From the definition in (9), considering the mutual independence of $\left\{\gamma_{r, k}^{\mathrm{DF}}\right\}_{k=1}^{N}$, and after some simple manipulation, $P_{\text {out }}^{\mathrm{DF}, 2}$ can be alternatively expressed as

$$
\begin{aligned}
P_{\mathrm{out}}^{\mathrm{DF}, 2}= & 1-\left(1-\prod_{k=1}^{N} \frac{P\left(\gamma_{r, k}^{\mathrm{DF}, 2}<\gamma_{\mathrm{th}}\right)}{P_{k}}\right) \\
& \times\left(1-P\left(\gamma_{d}^{\mathrm{DF}, 2}<\gamma_{\mathrm{th}}\right)\right) .
\end{aligned}
$$

By utilizing the known distribution of $\gamma_{1, k}$, it is easy to show that

$$
\begin{aligned}
P_{k} & =P\left(\frac{\gamma_{1, k}}{\gamma_{R, k}+1}<\gamma_{\text {th }}\right) \\
& =1-\int_{0}^{\infty} e^{-\gamma_{\mathrm{th}}(\gamma+1) / \rho_{1}} f_{\gamma_{R, k}}(\gamma) d \gamma .
\end{aligned}
$$

Substituting the PDF of $\gamma_{R, k}$ into (29) and using [9, Equation (3.351.3)], we have

$$
\begin{aligned}
P_{k}= & 1-\sum_{m=1}^{\varrho(\mathscr{A})} \sum_{n=1}^{\tau_{m}(\mathscr{A})} \mathscr{X}_{m, n}(\mathscr{A}) \rho_{R,[m]}^{-n} \\
& \times e^{-\gamma_{\mathrm{th}} / \rho_{1}}\left(\frac{\gamma_{\mathrm{th}}}{\rho_{1}}+\frac{1}{\rho_{R,[m]}}\right)^{-n} .
\end{aligned}
$$

Likewise, using the PDF of $\gamma_{2}^{\mathrm{DF}}$ and $\gamma_{D}$, we obtain

$$
\begin{aligned}
P\left(\gamma_{d}^{\mathrm{DF}, 2}<\gamma_{\mathrm{th}}\right)= & P\left(\frac{\gamma_{2}^{\mathrm{DF}}}{\gamma_{D}+1}<\gamma_{\mathrm{th}}\right) \\
= & 1-\sum_{m=1}^{\varrho(\mathscr{B}) \tau_{m}(\mathscr{B})} \sum_{n=1} x_{m, n}(\mathscr{B}) \rho_{D,[m]}^{-n} e^{-\gamma_{\mathrm{th}} / \rho_{2}} \\
& \times\left(\frac{\gamma_{\mathrm{th}}}{\rho_{2}}+\frac{1}{\rho_{D,[m]}}\right)^{-n} .
\end{aligned}
$$

Finally, substituting (30) and (31) into (28), we can obtain the closed-form expression for the outage probability of the DF relay system.

\section{Numerical Results}

In this section, we present some numerical results to verify the theoretical analysis. In Figures 2, 4, and 5, $N=2$,

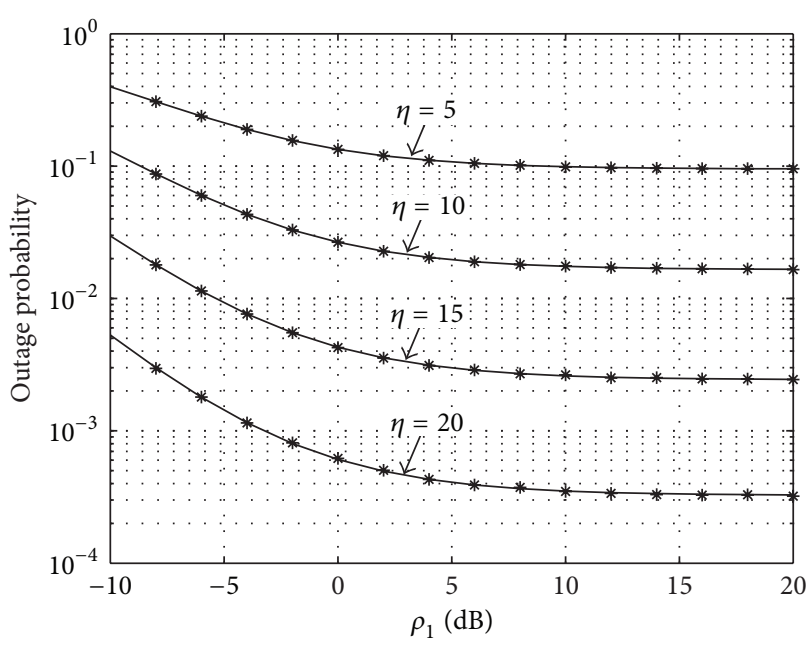

* Simulation

FIGURE 2: Outage probability of the AF relaying system versus SNR $\left(\gamma_{\text {th }}=0 \mathrm{~dB}\right)$.

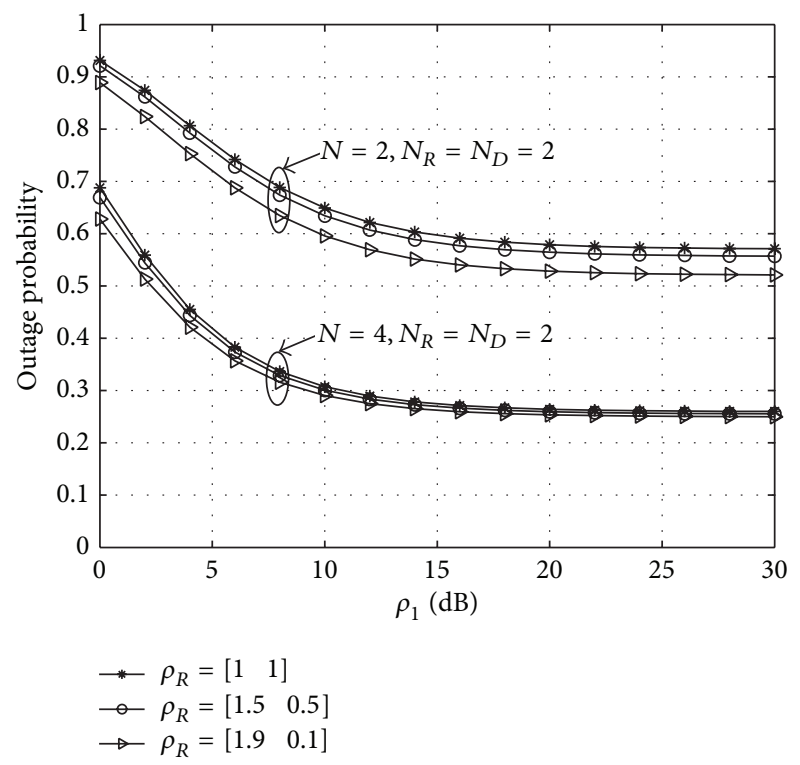

FIgURE 3: Impact of interference power distribution on the outage performance of the AF relaying system $\left(\rho_{2}=0 \mathrm{~dB}, \rho_{D}=\rho_{R}, \gamma_{\text {th }}=\right.$ $0 \mathrm{~dB})$.

$N_{R}=N_{D}=2, \rho_{1}=\rho_{2}, \mathbb{E}\left\{\gamma_{R}\right\}=\mathbb{E}\left\{\gamma_{D}\right\}, \rho_{R,[1]} / \rho_{R,[2]}=$ $\rho_{D,[1]} / \rho_{D,[2]}=1$, and the signal-to-interference ratio (SIR) is defined as $\eta=\rho_{1} /\left(\sum_{i=1}^{N_{R}} \rho_{R, i}+\sum_{j=1}^{N_{D}} \rho_{D, j}\right)$.

Figure 2 shows the outage probability of the dual-hop AF relaying system with the MRC/MRT scheme for different $\eta$. It is clear that all the analytical results are in exact agreement with the simulation results. Also, it is observed that in the high SNR range there is a floor effect, due to the impact of cochannel interference.

Figure 3 examines the impact of interference power distribution on the outage probability of the AF relaying system. 


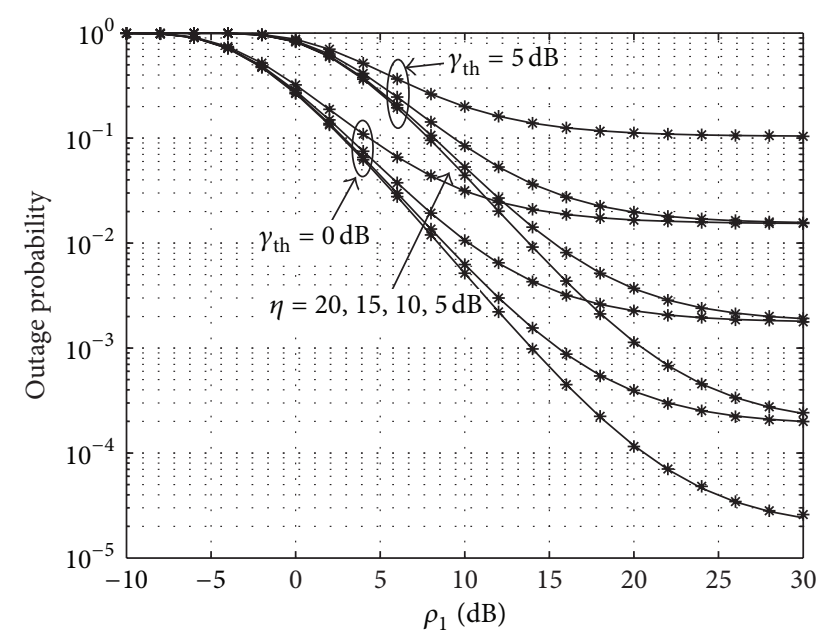

* Simulation

— Analysis

FIGURE 4: Outage probability of the DF relaying system with MRT versus SNR.

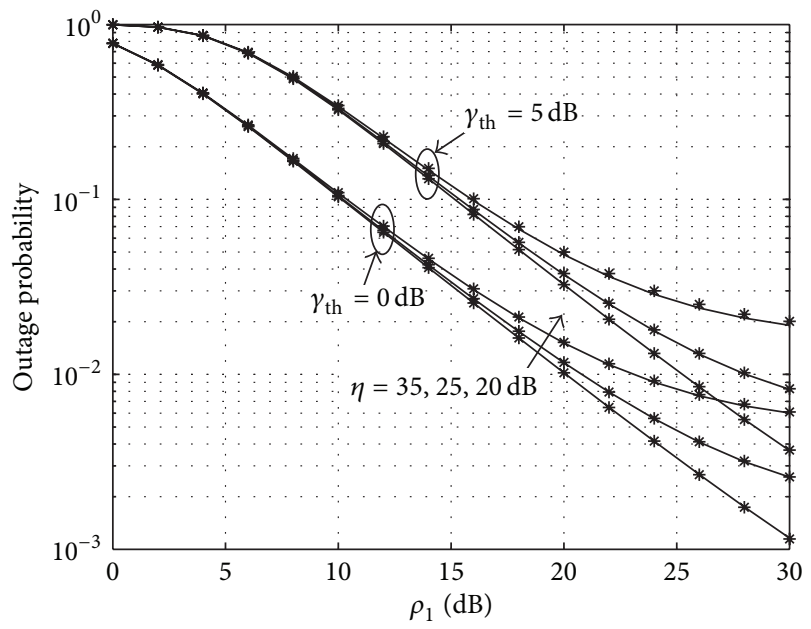

* Simulation

FIGURE 5: Outage probability of the DF relaying system without MRT versus SNR.

As illustrated, the equal interference power case yields the worst outage performance.

Figure 4 illustrates the outage probability of the dual-hop DF relaying system when maximal ratio transmission scheme is used at the relay. Also, it is clear that all the simulation results match the analytical results very well. As a result of the cochannel interference, the curves converge to a floor in the very high SNR range under the constraints of SIR $\eta$. The effect of threshold value $\gamma_{\text {th }}$ is also depicted, and we see that increasing $\gamma_{\text {th }}$ deteriorates the system performance as expected.

Figure 5 shows the outage probability of the DF relaying system without adopting any linear diversity combining

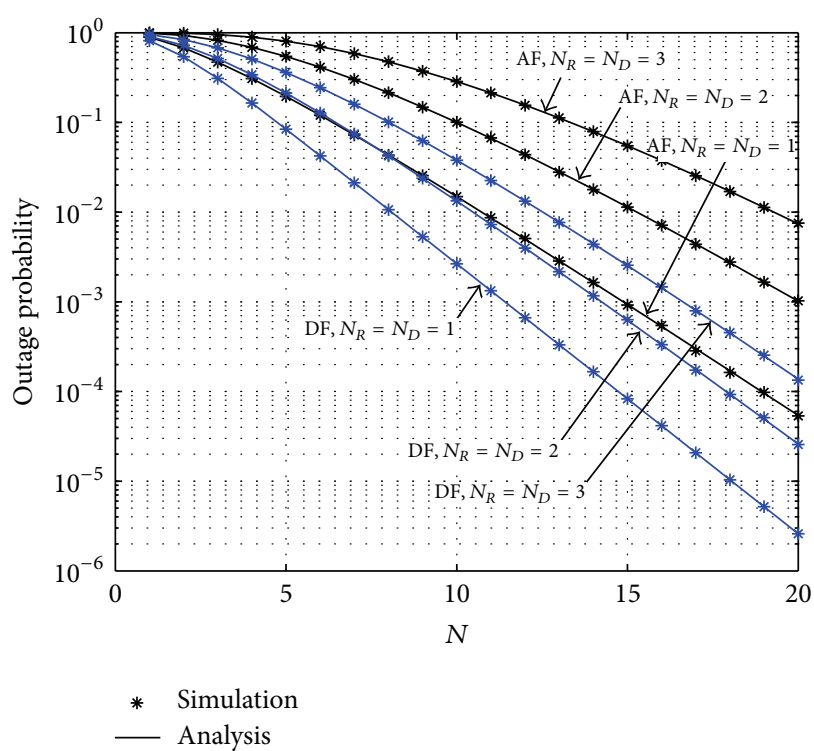

FIGURE 6: Comparison of AF relaying system with MRC/MRT and DF relaying system with MRT versus $N\left(\rho_{1}=\rho_{2}=0 \mathrm{~dB}, \rho_{R, i}=\right.$ $\left.\rho_{D, j}=0 \mathrm{~dB}, \forall i, j, \gamma_{\mathrm{th}}=0 \mathrm{~dB}\right)$.

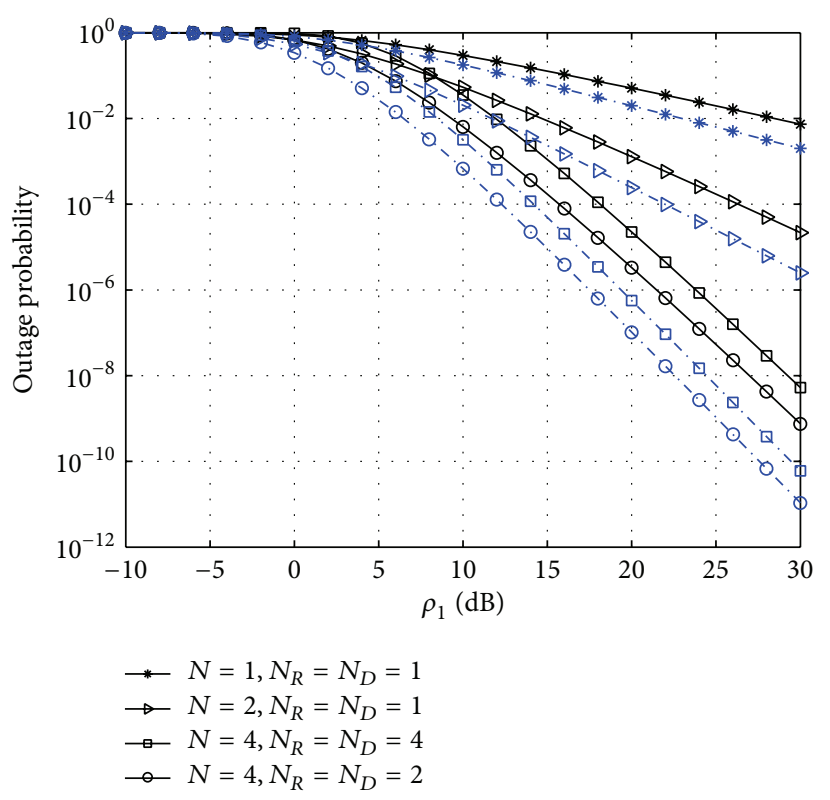

FIGURE 7: Comparison of AF relaying system with MRC/MRT and DF relaying system with MRT versus $\operatorname{SNR}\left(\rho_{1}=\rho_{2}, \rho_{R, i}=\rho_{D, j}=\right.$ $\left.0 \mathrm{~dB}, \forall i, j, \gamma_{\text {th }}=0 \mathrm{~dB}\right)$.

schemes for different $\eta$. From Figures 4 and 5, it is obvious that the DF relaying system can benefit from linear diversity combining scheme which has the capability of improving SINR.

Figure 6 compares the outage probability of the AF relaying system and DF relaying system as a function of the number of relay antennas $N$. We observe that increasing $N$ reduces the outage probability significantly, while increasing 
the number of interferers $N_{R}$ and $N_{D}$ degrades the outage performance.

Figure 7 compares the analytical results of AF relaying system with MRC/MRT scheme and DF relaying system with MRT scheme, using (22) and (26), respectively. The solid line denotes AF while the dashdot refers to DF. Because the DF relay fully decodes the source message $x$ and the noise at the relay is not amplified and forwarded to the destination, the outage performance of DF relaying system is superior to the AF system.

\section{Conclusion}

We investigated the outage performance of a dual-hop multiple antenna relaying system by taking into account the interference and noise at both the relay and destination. New exact closed-form expressions for the outage probability of the system employing AF and DF protocols were derived, which paved a fast and efficient way for understanding the effects of multiple antennas and interference on the system performance.

\section{Conflict of Interests}

The authors declare that there is no conflict of interests regarding the publication of this paper.

\section{Acknowledgments}

This work was supported in part by the National Natural Science Foundation of China under Grant 61322110, the National 863 Project of the Ministry of Science and Technology under Grants 2014AA01A705 and 2014AA01A706, and the National Science and Technology Major Project of the Ministry of Science and Technology under Grants 2012ZX03001030-004, 2013ZX03001020-002, 2013ZX03003009002, and 2013ZX03003016-005.

\section{References}

[1] W. Xu, J. Zhang, and P. Zhang, "Outage probability of two-hop fixed-gain relay with interference at the relay and destination," IEEE Communications Letters, vol. 15, no. 6, pp. 608-610, 2011.

[2] F. S. Al-Qahtani, J. Yang, R. M. Radaydeh, C. Zhong, and H. Alnuweiri, "Exact outage analysis of dual-hop fixed-gain AF relaying with CCI under dissimilar nakagami-m fading," IEEE Communications Letters, vol. 16, no. 11, pp. 1756-1759, 2012.

[3] H. Ding, C. He, and L. Jiang, "Performance analysis of fixed gain mimo relay systems in the presence of co-channel interference," IEEE Communications Letters, vol. 16, no. 7, pp. 1133-1136, 2012.

[4] C. Zhong, H. A. Suraweera, A. Huang, Z. Zhang, and C. Yuen, "Outage probability of dual-hop multiple antenna af relaying systems with interference," IEEE Transactions on Communications, vol. 61, no. 1, pp. 108-119, 2013.

[5] G. Zhu, C. Zhong, H. A. Suraweera, Z. Zhang, and C. Yuen, "Outage probability of dual-hop multiple antenna AF systems with linear processing in the presence of co-channel interference," IEEE Transactions on Wireless Communications, vol. 13, no. 4, pp. 2308-2321, 2014.
[6] K. Hemachandra and N. Beaulieu, "Analytical study of multiantenna relaying systems in the presence of co-channel interference," in Proceedings of the 76th IEEE Vehicular Technology Conference (VTC Fall '12), pp. 1-5, September 2012.

[7] K. S. Karthik and B. Ramamurthi, "A two-hop AF relaying scheme with interference suppression at the relay," IEEE Transactions on Vehicular Technology, vol. 63, no. 7, pp. 3469-3474, 2014.

[8] K.-C. Lee, C.-P. Li, T.-Y. Wang, and H.-J. Li, "Performance analysis of dualhop amplify-and-forward systems with multiple antennas and co-channel interference," IEEE Transactions on Wireless Communications, vol. 13, no. 6, pp. 3070-3087, 2014.

[9] I. S. Gradshteyn and I. M. Ryzhik, Table of Integrals, Series, and Products, Academic Press, San Diego, Calif, USA, 7th edition, 2007. 

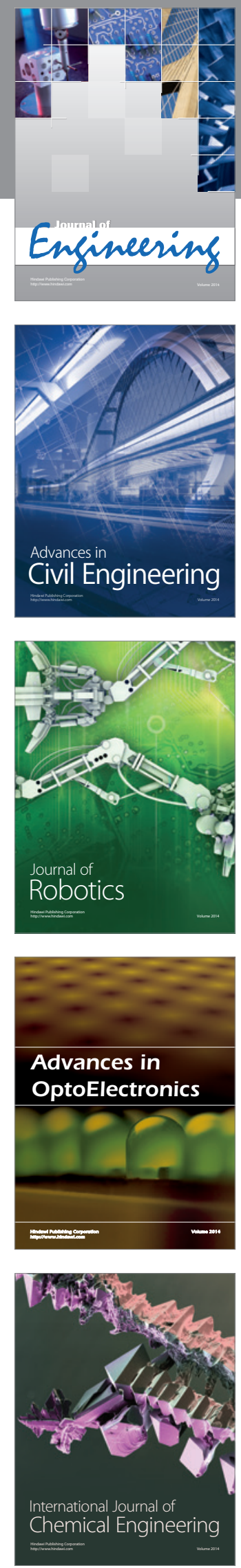

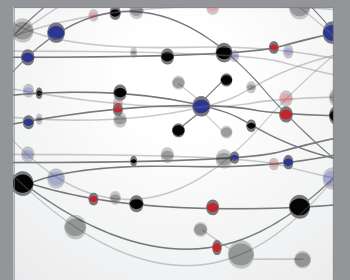

The Scientific World Journal
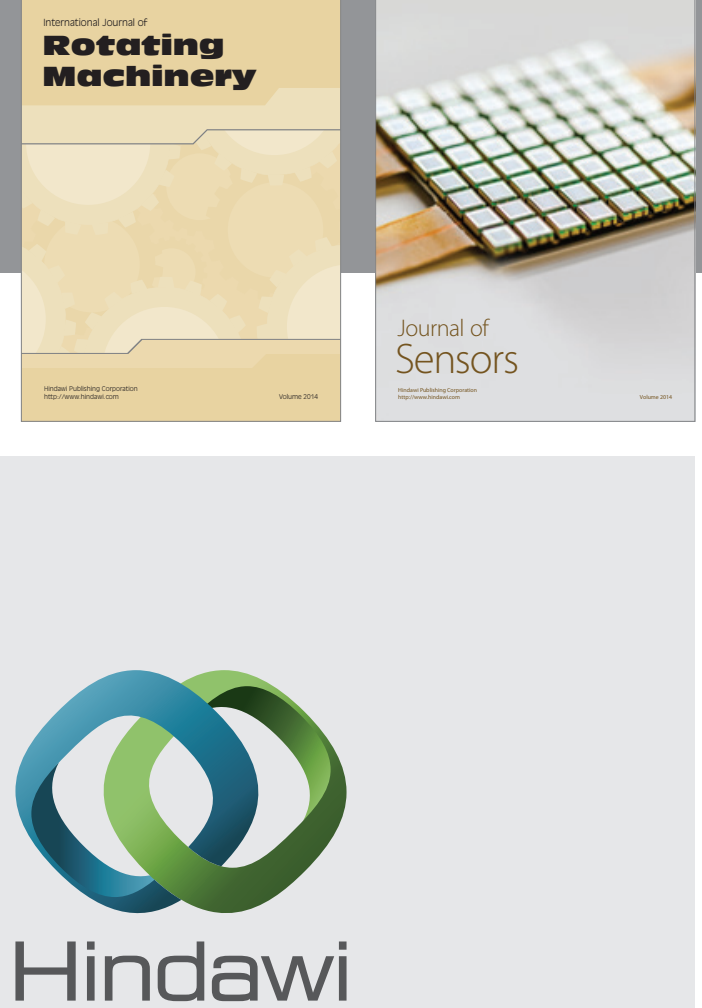

Submit your manuscripts at http://www.hindawi.com
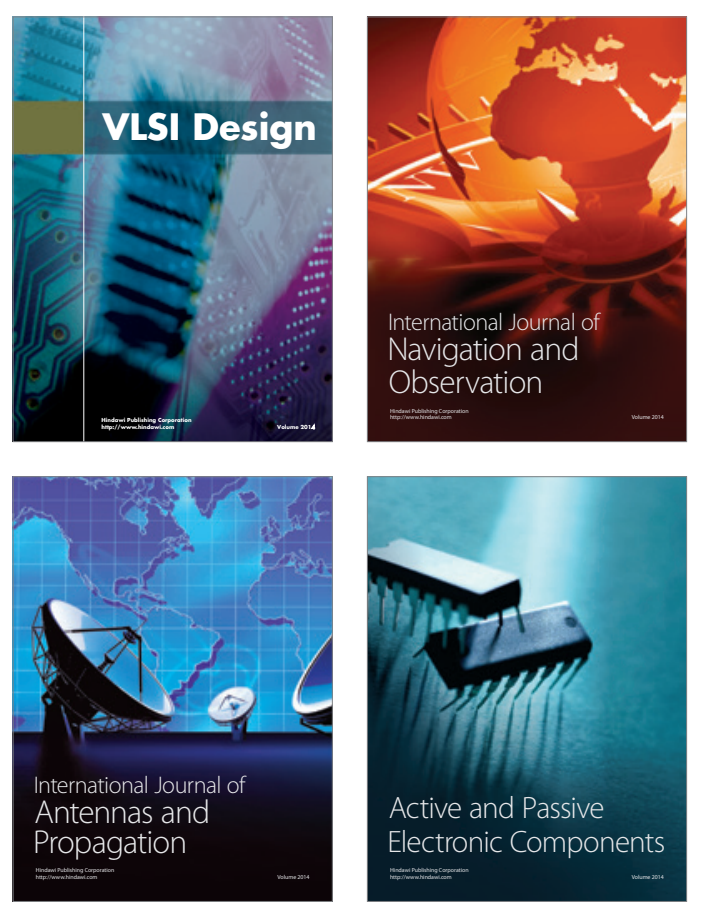
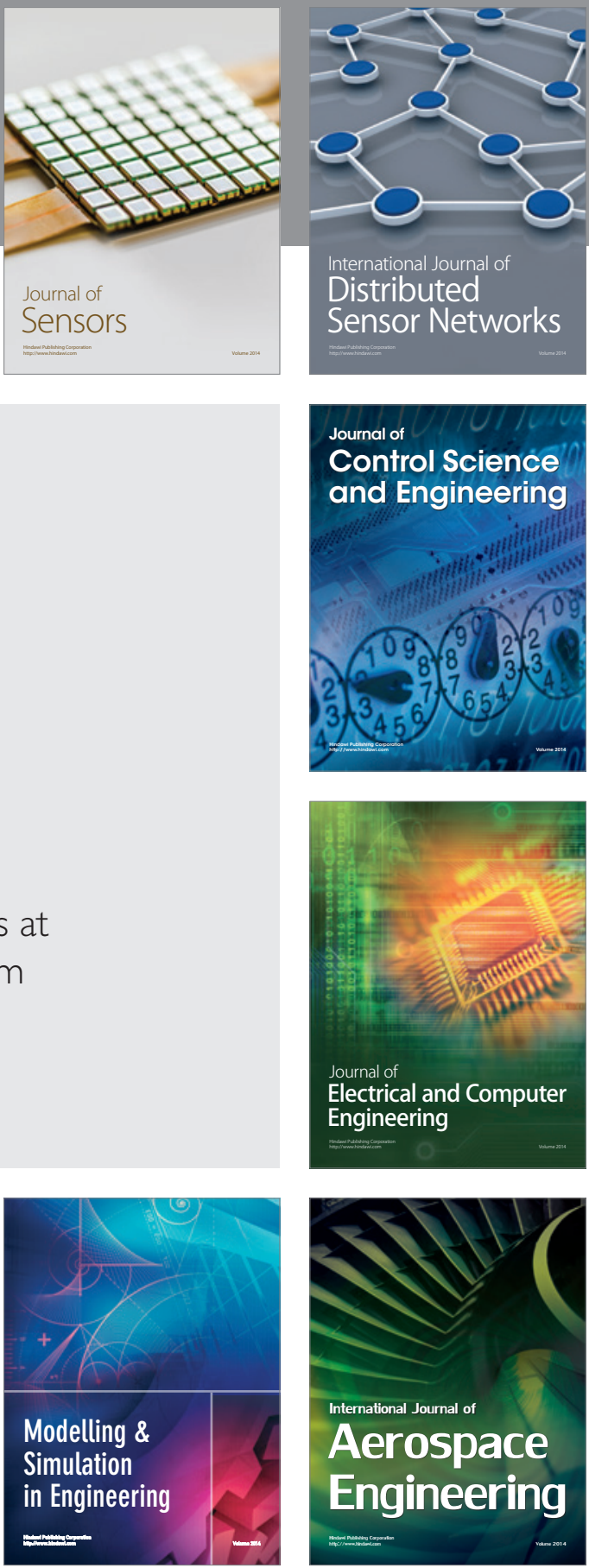

Journal of

Control Science

and Engineering
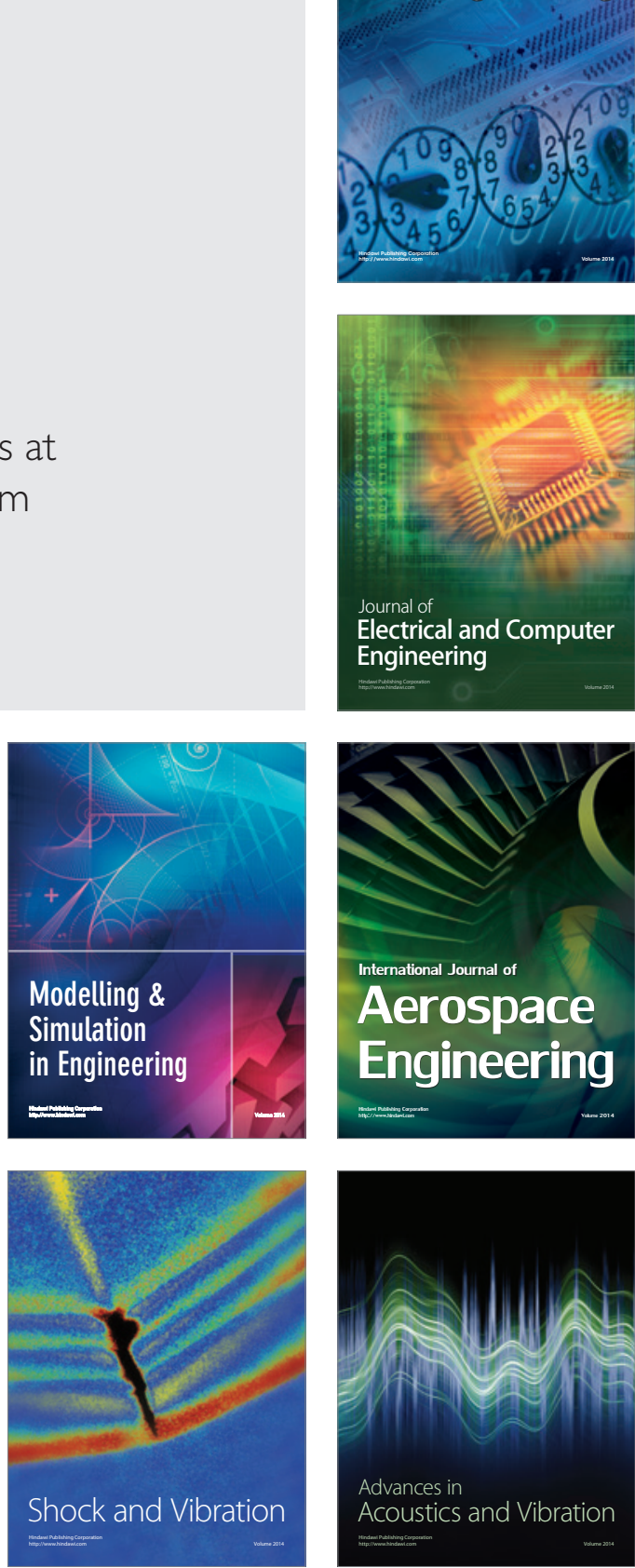